\title{
Wind-driven rain exposure and moisture damage risk of heritage facades
}

\author{
Aytac Kubilay, $^{\mathrm{a}, *}$, John Bourcet ${ }^{\mathrm{a}}$, Jessica Gravel ${ }^{\mathrm{b}}, \mathrm{Xiaohai}_{\text {Zhou }}^{\mathrm{a}}$, Dominique Derome ${ }^{\mathrm{b}}$, Jan Carmeliet ${ }^{\mathrm{a}}$ \\ ${ }^{a}$ ETH Zurich, Zurich, Switzerland \\ ${ }^{b}$ Université de Sherbrooke, Sherbrooke, Canada
}

\begin{abstract}
Accurate estimation of wind-driven rain (WDR) load on building facades is of paramount importance for the assessment of moisture-induced damage risks. Computational fluid dynamics (CFD) simulations of WDR are performed for the Parliament Buildings in Ottawa, Canada. Combined with a statistical analysis of the long-term local meteorological conditions, zones of facades receiving the highest amount of WDR are determined. Then, for critical locations on the facade, the Climatic Index is used as a metric, taking into account the total wetting load and total drying potential, in order to identify the façade locations and periods that show high risk of moisture damage.
\end{abstract}

Keywords: wind-driven rain; computational fluid dynamics; climatic index; durability

\section{Introduction}

Wind-driven rain (WDR) is a significant moisture source on building facades. The zones over the building envelope that are exposed to high amount of rain usually have a higher risk of deterioration due to moisture. Recently, Zhou et al. [1] proposed the Climatic Index as a metric to assess the risk of moisture-related damage based on wetting load due to WDR and evaporation potential. The Climatic Index allows for a fast evaluation of the risk of moisture-related problems. However, WDR load is typically calculated with semi-empirical models, which are prone to deficiencies and do not correctly capture the effects of local wind-flow features and building shapes [2]. In this study, we use computational fluid dynamics (CFD) simulations to obtain detailed distribution of surface wetting due to WDR on the Canadian Parliament Buildings located on Parliament Hill, Ottawa, Canada. CFD provides a significant variation of WDR intensity on the facades, which is not possible to capture with semi-empirical models. Then, we perform a statistical approach to find the critical facade locations, for which the Climatic Index is estimated.

\section{Methodology}

\subsection{Numerical simulations of wind-driven rain}

For the CFD simulations, a validated Eulerian multiphase model is used, which considers rain as continuum [3]. The numerical solver based on OpenFOAM, windDrivenRainFoam [4], solves the governing equations of rain, i.e. continuity and momentum, using the incompressible wind-flow field from steady Reynolds-averaged Navier-Stokes (RANS) calculations. The solver provides a database of WDR intensity on surfaces for reference values of wind direction, wind speed and rainfall intensity, based on the calculated rain velocity and rain phase fraction. This database for reference conditions can then be used to determine the actual WDR load by interpolation for given positions on building facade using local meteorological data. Past WDR research with CFD simulations considered mainly individual rain events [5]. However, for long-term data and large number of positions, this approach can become computationally expensive. Here, a statistical approach combines the numerical solutions and the meteorological data. For this, a Latin Hypercube Method (LHM) is used to select samples of wind direction, wind speed and rainfall intensity based on their frequency of occurrence [6]. Based on cumulative distribution functions, the generated samples are clustered around values of the highest frequency. The goal is to determine the zones of facades frequently receiving the highest amount of WDR.

\subsection{Climatic Index}

The orientation of the facade and the location on the facade strongly affects the WDR intensity. Similarly, it can also influence the evaporation potential. For example, a location can have both high exposure to WDR and solar radiation, or, in the opposite case, a location with high WDR exposure can have low evaporation potential, leading to moisture residing within the envelope for a longer duration. The Climatic Index is defined as the ratio between the annual wetting load and annual drying potential. The WDR load is obtained from the CFD simulations combined with the statistical approach explained in section 2.1. The evaporation

\footnotetext{
* Corresponding author: Aytaç Kubilay akubilay@ethz.ch
} 
ICMB21

potential is calculated based on the Penman equation [1]. A higher Climatic Index value corresponds to a higher moisture risk for the building envelope. Climatic Index shows an agreement with other indices that are based on hygrothermal simulations [1].

\section{Case study and results}

The computational domain for the CFD simulations is provided in Figure 1(a), which shows the explicitly-modeled buildings and terrain. A steep escarpment is located to the northwest of the Parliament Hill, descending to the Ottawa River. Figure 1(b) shows the normalized contours of wind speed for wind approaching from southeast. The resulting distribution of WDR intensity, normalized by horizontal rainfall intensity, is given for the central building in Figure 1(c) for a reference wind speed of $9 \mathrm{~m} / \mathrm{s}$ and a rainfall intensity of $1 \mathrm{~mm} / \mathrm{h}$. Severe WDR exposure is visible on the main tower and on the smaller towers to the north. Based on the 10-year meteorological data (2010-2020) from weather station Ottawa CDA RCS (Climate Ontario) and on the statistical LHM approach, numerical surface wetting in Figure 2(a) is obtained, where the locations with WDR exposure ratio larger than 0.15 are indicated in red. For the indicated positions P1, P2 and P3 facing east-northeast on the facade of one of the smaller towers, the Climatic Index values are shown in Figure 2(b). The values for position P1 are higher, indicating a more severe situation for higher locations on the façade. Furthermore, large differences in Climatic Index are observed for different years, showing 2010 being a more severe year mainly due to a larger amount of WDR events from east-northeast.

a)

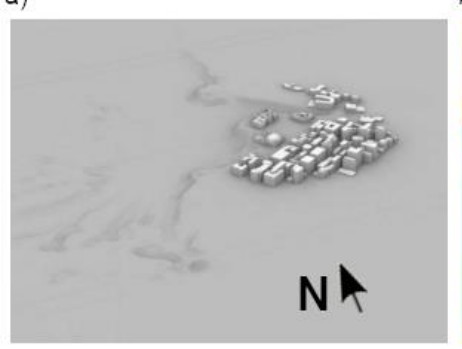

b)

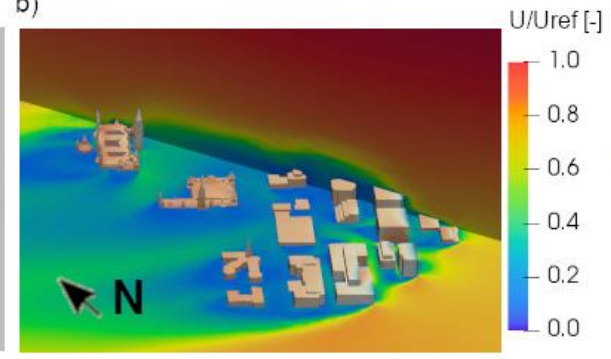

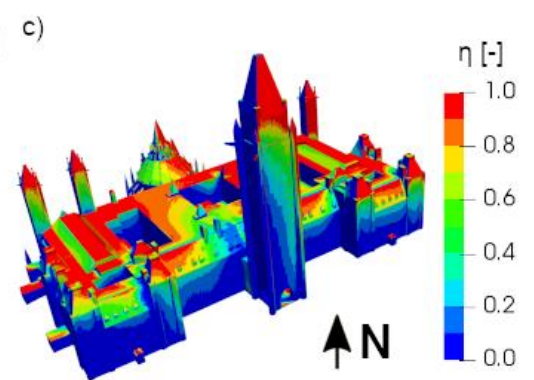

Figure 1. (a) Computational domain for the CFD simulations; (b) wind-flow field calculated for wind direction from southeast; (c) normalized surface wetting (catch ratio) for a wind speed of $9 \mathrm{~m} / \mathrm{s}$ and a rainfall intensity of $1 \mathrm{~mm} / \mathrm{h}$ [6].
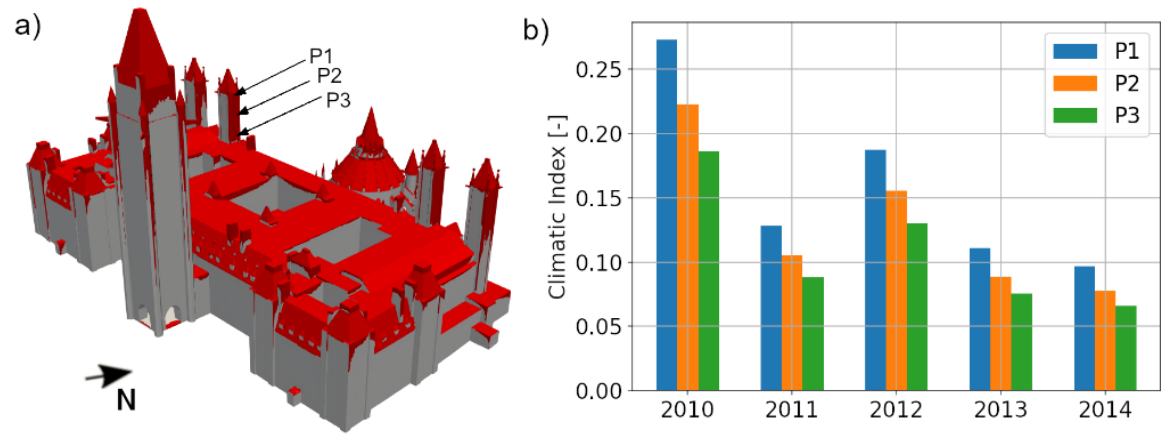

Figure 2. (a) Locations with higher WDR exposure; (b) comparison of the Climatic Index using WDR load from CFD simulations.

\section{Conclusions}

The combined approach of CFD with the Climatic Index helps to locate the critical zones/locations on building facade with high WDR exposure and assess the moisture damage risk. Further work is planned to perform hygrothermal simulations for those specific locations selected from CFD analyses and for the critical periods based on the climatic indices. Additionally, the impact of climate change will be investigated, which leads to higher temperatures on average and an increased amount of precipitation. The increase in temperatures will also convert some snowfall into rainfall, which will further increase the WDR exposure.

\section{References}

[1] Zhou X, Derome D, Carmeliet J. (2016). Robust moisture reference year methodology for hygrothermal simulations. Build. Environ., 110, 23-35.

[2] Kubilay A, Derome D, Blocken B, Carmeliet J. (2014). High-resolution field measurements of wind-driven rain on an array of low-rise cubic buildings. Build. Environ., 78, 1-13.

[3] Kubilay A, Derome D, Blocken B, Carmeliet J. (2014). Numerical simulations of wind-driven rain on an array of low-rise cubic buildings and validation by field measurements. Build. Environ., 81, 283-295.

[4] windDrivenRainFoam, An open-source solver for wind-driven rain based on OpenFOAM. https://carmeliet.ethz.ch/research/downloads/

[5] Blocken B, Carmeliet J. (2004). A review of wind-driven rain research in building science. J Wind Eng. Ind. Aerodyn., 92, 1079-1130.

[6] Bourcet J. (2020). Simulation of Wind-Driven Rain Exposure and Hygrothermal Analysis of Heritage Façades Using Future Climate Weather Data. MSc Thesis, ETH Zurich, Switzerland. 